Mots. Les langages du politique

\title{
La topique romantique dans les discours de l'écologie politique
}

Political ecology discourses and romanticism

El tópico romántico en el discurso de la ecología política

\section{Guillaume Carbou}

\section{(c) OpenEdition}

Journals

Édition électronique

URL : https://journals.openedition.org/mots/24494

DOI : $10.4000 /$ mots. 24494

ISSN : 1960-6001

Éditeur

ENS Éditions

Édition imprimée

Date de publication : 5 mars 2019

Pagination : 107-125

ISBN : 979-10-362-0130-1

ISSN : 0243-6450

\section{Référence électronique}

Guillaume Carbou, «La topique romantique dans les discours de l'écologie politique », Mots. Les

langages du politique [En ligne], 119 | 2019, mis en ligne le 01 janvier 2022, consulté le 23 avril 2022.

URL : http://journals.openedition.org/mots/24494 ; DOI : https://doi.org/10.4000/mots.24494

\section{(c) ENS Éditions}




\section{La topique romantique dans les discours de l'écologie politique}

L'objectif de cet article est de montrer que ce que nous appellerons la protestation romantique est l'un des moteurs idéologiques de l'écologie politique. Si d'autres auteurs ont déjà proposé des thèses similaires (voir l'entrée « romantisme » dans Bourg, Papaux, 2015; Audier, 2017), l'originalité de notre travail à cet égard repose sur le fait de montrer la vivacité de ce romantisme à la fois dans des textes fondateurs du corpus idéologique de l'écologie politique et dans des discours contemporains spontanés à propos des grands projets d'aménagement du territoire. De plus, en développant le répertoire topique du romantisme, nous proposons une grille de lecture permettant de repérer ce courant idéologique au sein de ce que la littérature décrit souvent et à juste titre comme la «nébuleuse» écologiste.

Cette problématisation appelle une précision à propos de ce que nous appelons romantisme. Le terme romantisme doit être pris ici non dans une acception simplement littéraire ou artistique, mais dans le sens politique que lui donnent notamment Robert Sayre et Michael Löwy : le romantisme est une protestation culturelle contre la modernité industrielle et capitaliste fondée sur le rejet du désenchantement du monde qu'elle produit (Löwy, Sayre, 1992). Nous tâcherons ici de donner une description analytique des principaux traits de l'expression politique du romantisme (sentiment de désenchantement du monde, critique de la modernité industrielle, rejet de la mécanisation et de l'artificialisation, déploration de l'immodération, contestation de la rationalité instrumentale, etc.).

Notre travail consistera alors à montrer la récurrence de cette topique d'une part chez des auteurs considérés comme fondateurs ou précurseurs de l'écologie politique (Ivan Illich, Bernard Charbonneau, André Gorz, Murray Bookchin, Arne Naess et Henry David Thoreau) et d'autre part dans un corpus de commentaires d'internautes protestant contre des projets d'aménagement du territoire sur des sites de presse en ligne. L'objectif n'est évidemment pas de définir ici ce qu'est le romantisme, ce qui n'aurait aucun sens, et encore moins ce 
qu'est l'écologie, ce qui n'en aurait pas plus. Il s'agit en revanche de montrer que le romantisme tel que nous le décrivons est présent dans nombre de discours considérés comme écologistes et en constitue un moteur important. Ce n'est bien sûr pas le seul, car il existe bien des raisons d'être écologiste : on peut par exemple être écologiste par amour d'une Nature sacralisée ou encore par utilitarisme en souhaitant se protéger des conséquences négatives d'activités potentiellement dangereuses. Ces moteurs idéologiques, bien éloignés du romantisme, se mélangent souvent dans les attitudes et les discours, ce qui peut rendre l'analyse difficile. Il n'en reste pas moins que les traits caractéristiques du romantisme y apparaissent avec force et c'est ce que nous tâcherons de montrerici.

Le plan de notre article est le suivant. Dans un premier temps, nous proposerons une clarification théorique sur la manière dont nous approchons l'espace public discursif. Dans un second temps, nous décrirons la forme idéologique romantique telle que nous la concevons et tâcherons de montrer sa présence dans un corpus de textes influents de l'écologie politique. Enfin, nous montrerons que sous des formes plus spontanées, mais non moins évidentes, les mêmes idées parcourent les discours de contestation d'internautes opposés à de grands projets d'aménagement du territoire. Notre conclusion insistera sur une des caractéristiques les plus remarquables de cette écologie romantique : la quasi-absence de référence à la nature dans son armature idéologique. Nous soulignerons par ailleurs l'intérêt de mettre au jour la forme idéologique romantique : celle-ci, bien que peu ou pas conscientisée et surtout jamais nommée, apparaît pourtant particulièrement agissante dans la dynamique de la pensée et de l'action écologiques contemporaines.

\section{Espace public sémiotique et analyse topique}

Notre travail consiste à rechercher des formes culturelles agissantes dans l'espace public à partir d'une analyse topique. Cette ambition repose sur une conception sémiotique de l'espace public et de la culture pour laquelle

il s'agit de substituer à la subjectivité individuelle, posée comme support de l'action, de la connaissance et du sens, un fondement d'ordre public, c'est-à-dire un cadre institué, partagé, connu en commun et accessible à quiconque a une compétence de membre d'une collectivité. [...] [Ce] fondement public [...] est le plus couramment défini en termes de jeux de langage, de formes de vie, de formes symboliques, de Lebenswelt, les dénominations variant suivant les approches. (Quéré, 1992, p. 87)

Notre objectif est donc ici de décrire, au sein des phénomènes culturels dynamiques qui ont lieu dans l'espace public sémiotique, une forme spécifique, relativement cohérente et stable, que nous nommons romantisme. Ce 
romantisme dont nous donnerons plus loin les traits caractéristiques n'est par ailleurs pas un objet du monde, mais bien un idéaltype (au sens de Max Weber) à visée heuristique.

Ainsi, chercher à décrire des formes idéologiques circulant dans l'espace public, c'est reconstruire des idéaltypes sans les hypostasier, à partir de fragments épars circulant dans l'interdiscours. Plus précisément, nous nous inscrivons dans le cadre théorique du «discours social» développé par Marc Angenot (2006) et adoptons une démarche d'analyse topique inspirée par le même auteur (Angenot, 1982) et que nous avons développée ailleurs (Carbou, 2015). Dans cette perspective, la tâche de l'analyste est de rassembler le répertoire topique des formes idéologiques qui se construisent et se diffusent dans l'espace public afin d'en exprimer la forme la plus idéaltypique possible. Dans cette entreprise, le concept de topos prend une acception très large. Il s'agit de l'ensemble des thèmes, idées, valeurs, fondements épistémiques qui caractérisent une forme de pensée.

Ces considérants théorico-méthodologiques étant posés, nous allons, dans la section suivante, dresser le répertoire topique du romantisme tel que nous l'entendons et nous montrerons comment chacun de ses pivots est également central dans le discours de plusieurs penseurs de l'écologie.

\section{La forme romantique et sa prégnance dans le corpus idéologique des penseurs de l'écologie}

Nous étudierons ici un corpus de six textes considérés comme fondateurs ou

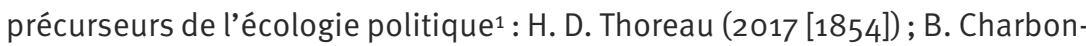
neau (1937); M. Bookchin (1976 [1964]); I. Illich (1973); A. Gorz (1977); et A. Naess (2008 [1989]). Il ne s'agira évidemment pas de dire que les auteurs que nous étudions sont romantiques, les philosophies de certains, prises dans leur globalité, peuvent même s'éloigner de la forme romantique, mais il n'en reste pas moins qu'elles s'appuient sur certains éléments de celle-ci et participent donc à la nourrir et à la renforcer. Précisons bien que nous concevons le romantisme comme une forme culturelle transdiscursive qui peut s'exprimer sur des thématiques et dans des arènes diverses : nous entendons montrer ici qu'elle s'épanouit dans les discours écologistes, sans leur être ni exclusive ni nécessaire.

Le romantisme tel que nous l'entendons prend appui sur les travaux de M. Löwy et R. Sayre. Ces deux auteurs ont consacré une large partie de leur travail à étudier et à caractériser le romantisme politique à partir du constat que celui-ci «représente [...] un véritable continent oublié qui échappe aux grilles

1. Ceux-ci sont en effet cités dans la grande majorité des travaux de synthèse ou historiques sur la pensée écologique (Flipo, 2014). 
habituelles dans les sciences humaines» (Sayre, Löwy, 1983). Pour en donner une expression synthétique,

le romantisme doit être conçu comme une vision du monde [...] dont la caractéristique quintessencielle est la protestation culturelle contre la civilisation capitaliste moderne au nom de certaines valeurs du passé. Ce que le romantisme refuse dans la société industrielle/bourgeoise moderne, c'est avant tout le désenchantement du monde [...], c'est le déclin ou la disparition de la religion, de la magie, de la poésie, du mythe, c'est l'avènement d'un monde entièrement prosaïque, utilitariste, marchand. Le romantisme proteste contre la mécanisation, la rationalisation abstraite, la réification, la dissolution des liens communautaires et la quantification des rapports sociaux. (Löwy, Blechman, 2004, p.3)

La forme culturelle romantique est évidemment dynamique et multiforme, et M. Löwy et R. Sayre proposent d'en circonscrire, à toute fin heuristique, plusieurs grandes tendances, du conservatisme le plus réactionnaire au progressisme utopiste en passant par des formes de critique désabusées et fatalistes (Sayre, Löwy, 1984). Nous nous concentrerons ici sur le « romantisme révolutionnaire », qui entend proposer les contours d'une utopie inspirée d'un passé idéalisé mais résolument tournée vers l'avenir. Le romantisme révolutionnaire enregistre les méfaits d'une modernité oublieuse de l'épanouissement humain et imagine une société fondée sur des valeurs qualitatives plutôt que quantitatives.

Les écologies qui portent la topique romantique font particulièrement écho à cette forme de romantisme. Nous en proposons ci-dessous une présentation analytique ${ }^{2}$ en insistant sur ses traits caractéristiques et sur leur présence chez les auteurs que nous avons sélectionnés.

Pour ce faire, nous découpons la topique romantique en cinq grands axes qui se connectent selon la logique suivante :

1) Le romantisme prend sa source dans une réaction au sentiment de désenchantement du monde que produit la société moderne. Ce désenchantement peut être analysé comme le résultat de quatre types d'aliénation des individus (économico-politique; philosophique; épistémique; technique). 2a) L’individu moderne est aliéné par le système économique et politique, car la gestion centralisée, utilitariste et bureaucratique le réduit à un simple rouage. 2 b) Le romantisme oppose à ce mode de vie un mode de vie qui respecte l'autonomie créatrice des individus en étant «à taille humaine». 3a) La philosophie moderne de la performance généralisée est une seconde source de contention des individus qui s'aliènent à une vaine quête du «toujours plus». 3b) Le romantisme y oppose les bénéfices d'une vie sage, sobre, harmonieuse et joviale. 4a) L'aliénation épistémique est quant à elle produite

2. Nous insisterons ici sur cinq axes topiques caractéristiques. Pour une présentation plus détaillée, intégrant les spécificités discursives du romantisme, on pourra se reporter à Carbou (à paraître). 
par l'hégémonie de la raison scientifique sous la forme de la rationalisation abstraite et mathématique du monde. Celle-ci aliène les individus en remplaçant l'expérience subjective du monde par un rapport entièrement analytique et prosaïque. 4b) Le romantisme oppose à la rationalisation abstraite un réenchantement par le biais de la spiritualité et revendique, contre la seule raison mathématique, une raison philosophique capable de saisir la diversité et la complexité du monde. 5a) Enfin, le progrès technique et la technicisation de la société asservissent l'individu et l'empêchent d'avoir la maîtrise de ses conditions d'existence. 5 b) Le romantisme propose une réorientation de l'usage de la technologie en la subordonnant à l'émancipation individuelle et collective. Ci-après, nous développons ces cinq axes topiques et montrons qu'ils se retrouvent de manière saillante chez l'ensemble des auteurs de notre corpus.

\section{1) Désenchantement et aliénation}

Le romantisme est initialement un sentiment d'inadéquation fondamentale entre les conditions de la vie moderne et les conditions de l'épanouissement humain. Comme sa version littéraire, le romantisme politique insiste sur le nécessaire respect de la personne humaine dans sa singularité et son autonomie. La réification ou l'aliénation, en tant que dépossession des individus de leur libre arbitre et de leur libre activité, représentent ainsi son ennemi principal.

Cette posture se retrouve au fondement des textes de notre corpus. Les auteurs écologistes versant dans l'anarchisme (M. Bookchin ou H. D. Thoreau par exemple) en sont particulièrement représentatifs. Le problème du monde n'est pour eux ni les inégalités en tant que telles, ni les nuisances directes que peut entraîner telle ou telle technique, ni même la destruction de la nature, mais bien la réduction des possibilités d'épanouissement de la personne humaine. L'écologie d'inspiration anarchiste se soulève ainsi contre l'aliénation qu'elle attribue au centralisme technocratique ou à l'utilitarisme marchand.

Avec des origines idéologiques différentes, le personnalisme de B. Charbonneau présente une posture très proche lorsqu'il revendique «la prééminence de la personne concrète et des communautés sur les masses, [et une] hostilité commune contre le rationalisme bourgeois [...] la grande ville, la bureaucratie, l'oppression de l'argent et de l'État» (Charbonneau, 1937, p. 58). L'ouvrage d'I. Illich est quant à lui une charge contre « une société qui [...] mutile de façon intolérable l'autonomie de la personne» (Illich, 1973, p. 31). Chez A. Naess, qui cite abondamment Spinoza, la société doit permettre à chacun de s'épanouir, de persévérer dans son être (le fameux right to blossom de l'écologie profonde), idéal rendu impossible par l'enfermement de l'individu dans la société industrielle.

Les causes de l'aliénation de l'individu moderne sont multiples et entrelacées, mais nous pouvons en distinguer quatre principales, avec leurs solutions romantiques. 


\section{2a) Les deux premières mamelles de l'aliénation: utilitarisme et bureaucratie}

Un puissant facteur de désenchantement est pour le romantisme l'avènement d'un monde entièrement prosaïque où l'action humaine est régulée par une pensée gestionnaire et utilitariste. La pensée utilitariste empêche d'éprouver un rapport complet au monde puisque seuls des objectifs, fixés à l'avance et considérés de manière réductrice, comptent pour évaluer et contrôler l'activité humaine. La forme gestionnaire, entendue comme la rationalisation de l'action collective, et qui culmine dans la bureaucratie, provoque quant à elle l'atomisation des individus : ils sont cantonnés à des tâches spécialisées, répétitives, sans pouvoir d'invention et de décision, et souvent sans possibilité de saisir le sens global de leur action individuelle.

Le corpus de discours écologistes que nous étudions ici se fonde également en grande partie sur la critique de la bureaucratie et de l'utilitarisme. Tous (mis à part H. D. Thoreau pour des raisons historiques) pointent d'ailleurs du doigt les dérives «technofascistes» (Gorz, 1977) auxquelles pourrait mener une écologie centralisée et utilitariste fondée sur la seule protection de l'environnement. Plus généralement, la bureaucratie, en tant qu'elle refuse toute liberté et tout pouvoir de décision à la personne particulière, suscite une vive critique. Chez B. Charbonneau, bien sûr, dans une optique personnaliste : «toutes les révolutions modernes se traduisent par l'oppression plus grande d'une société identifiée à l'État sur les personnes» (Charbonneau, 1937, p. 21). A. Gorz et I. Illich, quant à eux, se font les contempteurs de l' «esclavage» produit par la «mégamachine»: «la survie dans l'équité ne sera ni le fait d'un oukase des bureaucrates ni l'effet d'un calcul des technocrates» (Illich, 1973, p. 33). Tous rejettent un système qui met sous l'éteignoir l'autonomie créatrice et libératrice de l'individu : "les techniques bureaucratiques de gestion de la société tendent à remplacer les méthodes humanistes » (Bookchin, 1976, p. 80).

\section{2b) Pour une société «à taille humaine»}

Cette critique des effets d'un système gestionnaire centralisé mène le romantisme à revendiquer des formes de vie à petite échelle. En effet, une vie «à taille humaine » doit permettre aux individus d'avoir la main sur leurs conditions d'existence. C'est cette nécessité qui amène nombre de romantismes à idéaliser la vie communautaire traditionnelle de groupes sociaux capables de s'autogérer.

La thématique de l'autogestion et sa critique du gigantisme sont très présentes dans le corpus anarcho-écologiste (le municipalisme libertaire de M. Bookchin en est un parangon, tout comme le style de vie communau- 
taire chez A. Naess). H. D. Thoreau radicalise ce processus en recherchant une (quasi-)autonomie individuelle. B. Charbonneau ne cesse quant à lui de vanter la qualité des relations qui s’instituent dans les communautés réduites :

ce qui importe, ce n'est pas l'armature administrative : grands rassemblements, hiérarchie, congrès, mais le petit groupe : la patrouille, la bande, le camp; chacun de ces groupes vit d'une vie très particulière. (Charbonneau, 1937, p. 48)

Plus encore, les philosophes de notre corpus partagent avec la critique romantique de la modernité une apparente ambiguïté. D’une part, ils critiquent le fait que la modernité marchande entraîne la dissolution des liens sociaux, le déracinement, la perte des traditions, etc. Et d'autre part ils déplorent la disparition de la liberté individuelle produite par la bureaucratie et la société de contrôle étatique. Ce qui semble être un paradoxe entre des valeurs plutôt conservatrices d'un côté et plutôt libérales de l'autre relève en fait d'une conception dialectique de l'être humain : l'épanouissement individuel recherché n'est pas tant l'épanouissement d'un je radicalement individuel mais d'un soi toujours déjà social. Celui-ci s'épanouit non dans le vide mais dans l'harmonie avec une totalité communautaire ou mieux, universelle (la première proposition risquant de mener à un organicisme fascisant). Cette conception dialectique qui fait de la personne le tiers médiateur entre l'individu et la société est particulièrement marquée dans le personnalisme de B. Charbonneau - «la recherche d'une vie naturelle est à la fois une recherche de solitude et une recherche de la camaraderie» (Charbonneau, 1937, p. 47) -, mais également très présente chez I. Illich :

un nouveau système de production doit retrouver la dimension personnelle et communautaire. La personne, la cellule de base conjuguent de façon optimale l'efficacité et l'autonomie : c'est seulement à leur échelle que se déterminera le besoin humain dont la production sociale est réalisable. (Illich, 1973, p. 27)

Elle est aussi centrale dans la conception du «Soi » universel d'A. Naess ou dans le «cosmos libertaire» d'H. D. Thoreau (pour un développement, voir Guest, 2012).

\section{3a) L'aliénation à la croissance perpétuelle}

La décroissance de la taille des systèmes sociaux s’inscrit dans une tendance plus générale du romantisme à protester contre tout ce qui peut être vu comme un excès. L'avidité, les ambitions, la volonté de puissance ou de performance lui apparaissent comme autant de facteurs d'aliénation. Vouloir toujours plus, c'est s'arrimer à une quête sans fin.

La critique du «trop» est présente dans notre corpus de manière évidente : les auteurs que nous étudions peuvent tous être inscrits dans le courant de la décroissance ou de la simplicité volontaire. Les plus économistes (M. Bookchin, 
A. Gorz, I. Illich) portent un propos plutôt technique sur les intérêts de la limitation, les plus spiritualistes (H. D. Thoreau, A. Naess, voire B. Charbonneau) insistent quant à eux sur les vertus morales de l'ascétisme : «Ne cherchez pas si avidement à vous développer, à vous soumettre à de nombreuses influences pour être joué; ce n'est que dissipation » (Thoreau, 2017, p.360). Tous portent une critique du consumérisme et du productivisme. Les longs passages ironiques d'H. D. Thoreau sur les ornementations bourgeoises entrent dans cette ligne, et plus encore ses appels au ralentissement des modes de vie : «Pourquoi devrionsnous vivre dans une telle hâte en gaspillant nos vies?» (Ibid., p. 108). Le concept de "seuil de contre-productivité», au-delà duquel l'augmentation de quelque chose devient néfaste, proposé par I. Illich et largement repris par A. Gorz, s’inscrit de la même manière dans une optique modératrice. Les appels d'l. Illich à la sage modération sont également légion : «l'homme retrouvera la joie de la sobriété et de l'austérité en réapprenant à dépendre de l'autre, au lieu de se faire l'esclave de l'énergie et de la bureaucratie toute puissante» (Illich, 1973, p. 33).

\section{3b) Sobriété heureuse et harmonie comme remèdes à l'avidité}

L'alternative romantique à la vaine course au «toujours plus» est double. Elle se situe d'une part dans la recherche d'une sobriété heureuse, dans la possibilité de jouir des plaisirs simples de la vie. Ainsi des remarques de B. Charbonneau : «la vie en montagne nous apprend que le bonheur ne s'établit pas, mais qu'il est attaché à la peine et qu'au-delà d'un certain confort, toutes les sources de joie vraiment humaines sont taries» (Charbonneau, 1937, p.46); et bien sûr d'H. D. Thoreau qui entend dépouiller l'existence humaine pour jouir de ses fondements les plus authentiques : «les plus sages ont toujours vécu une vie plus simple et dépouillée que les pauvres» (Thoreau, 2017, p. 23). D'autre part, l'ambition romantique n'est pas celle d'une amélioration perpétuelle, mais d'une intégration dans un système équilibré. Il s'agit de rechercher l'harmonie. La pensée romantique est une pensée de la totalité, du système, qui se retrouve aisément dans les philosophies écologistes : cela est très clair dans les spiritualités spinozisto-bouddhistes d'A. Naess ou d'H. D. Thoreau, mais tout autant chez I. Illich (chapitre "L'équilibre» : Illich, 1973, p. 72-113) ou dans l'anarchisme de M. Bookchin :

Si une communauté écologique se réalise jamais, la vie sociale suscitera une diversification subtile du monde humain comme du monde naturel et les réunira en un tout harmonieux et équilibré. (Bookchin, 1976, p. 84)

\section{4a) Contre la dictature de la Raison}

Le romantisme s’apparente au «crépuscule des Lumières» (Gusdorf, 1982). Il s’élève contre la tendance hégémonique de la raison scientifique cartésienne 
sur la vie des sociétés humaines. Il s'agit là d'un corollaire de l'épistémologie romantique évoquée ci-dessus : en tant que projet de penser la totalité et les équilibres, le romantisme ne peut voir que réductionnisme dans les sciences logico-mathématiques. Plus prosaïquement, la rationalisation abstraite s'oppose frontalement à la passion romantique pour la profondeur et la diversité de l'expérience humaine.

On retrouve cette idée de manière explicite chez $M$. Bookchin pour qui la

suspicion jetée sur la science et la méthode scientifique, est loin d'être infondée. «Bien des gens sensibles, en particulier parmi les artistes craignent que la science ne flétrisse et n'avilisse les choses, qu'elle ne les sépare au lieu de les relier, faisant ainsi œuvre de mort plutôt que de création », écrit Abraham Maslow. (Bookchin, 1976, p.71)

Et de même chez B. Charbonneau :

notre raison ne peut réduire l'objet naturel à un scénario [...] «l'ai réappris que l'homme est un être fini, sa raison ne peut saisir qu'une infime part du réel [...].» (Charbonneau, 1937, p.47)

Tout calcul coût/bénéfice, toute tentative de maîtrise de la complexité du monde à partir d'indicateurs chiffrés ou de catégories logiques sont ainsi vus comme une réduction inopérante et destructrice. Nous l'avons vu plus haut, les auteurs de notre corpus portent une vive critique contre les systèmes gestionnaires rationalisés. Un des traits les plus vilipendés est leur « obsession de la quantification» (Illich, 1973, p.15) dont les conséquences sont, encore une fois, l'aliénation des individus : «En réalité, l'industrialisation des besoins réduit toute satisfaction à un acte de vérification opérationnelle, remplace la joie de vivre par le plaisir d’appliquer une mesure.» (ibid., p. 41).

\section{4b) De la raison au raisonnable}

Le constat du désenchantement rationaliste pousse le romantisme à proposer diverses voies de réenchantement : poésie, exaltation des valeurs communautaires, réhabilitation de l'ésotérique, du mythe ou du religieux. C'est cette posture qui lui vaut généralement les accusations d'irrationalisme et/ou d'inclinations fascisantes (Audier, 2017).

La question de l'enchantement dans le romantisme est donc complexe et mériterait des développements que nous ne pouvons réaliser ici. Nous nous contenterons de remarquer que si tous nos auteurs portent des charges violentes contre la religion pour son caractère potentiellement aliénant (ce fondement libertaire sans équivoque doit ainsi éloigner toute suspicion de fascisme latent), nous avons montré que leur critique de la modernité capitaliste se fondait sur le constat de la déshumanisation qu'elle produit (disparition 
des valeurs, du sens, des relations profondes sous l'utilitarisme généralisé). L'enchantement du monde éco-romantique est ainsi à trouver dans la profondeur et la diversité du lien, toujours créatif et dynamique (donc non identitaire), que l'être humain peut nouer avec son environnement (dont ses semblables) et qui ne peut être réduit à un simple calcul ou à une expression formelle. Cette revendication peut prendre plusieurs formes : demande de «sens»; valorisation des relations humaines et de la camaraderie; appel à l'oisiveté et à la contemplation; défense de la diversité des modes et des formes de vie, etc. Par ailleurs, il faut noter la part importante de références, sinon religieuses, du moins spirituelles dans notre corpus d'écologie politique : I. Illich était prêtre - certes hétérodoxe - et B. Charbonneau, tout en éreintant l'institution religieuse, s'inscrira dans la mouvance personnaliste d'Emmanuel Mounier et de la revue Esprit avec Jacques Ellul. Nous pouvons également souligner le transcendantalisme d'H. D. Thoreau qui, fustigeant la religion de son temps pour son rigorisme, entend permettre une forme d'éveil dans la vie spartiate. Rappelons que l'œuvre d'H. D. Thoreau est traversée d'allusions ou d'emprunts directs aux spiritualités bouddhistes/hindouistes. A. Naess revendique quant à lui tout un corpus bouddhiste.

Par ailleurs, cette défense du spirituel au sens très large, concomitant d'une déploration des méfaits de l'hyperrationalisme attribué aux Lumières, n'est en aucune façon irrationaliste. Le romantisme ne s'oppose pas à la rationalité tout court, mais soit à la rationalité instrumentale en particulier, soit à la tendance de celle-ci à se vouloir applicable à toutes les sphères de la vie (Löwy, Sayre, 2010). Plus largement, le romantisme revendique la possibilité, pour guider les actions humaines, d'une forme de pensée qui ne soit ni rationnelle ni irrationnelle mais bien raisonnable, pour reprendre la célèbre distinction de Chaïm Perelman (Angenot, 2012). Une raison prudentielle, casuistique, philosophique, aux côtés d'une raison mathématique, logique et apodictique. M. Bookchin, appelant à une science écologique, explicite bien cette perspective :

Pénétrées pour l'essentiel d'un esprit fonctionnel et instrumental, [les] sciences qui autrefois menaçaient de rompre les chaînes de l'homme, servent aujourd'hui à les perpétuer, à les dorer. [...] Il existe pourtant une science qui peut encore retrouver et même surpasser la valeur libératrice des sciences et des philosophies d'autrefois. On la dénomme de façon relativement vague «écologie». (Bookchin, 1976, p. 96)

\section{5a) Critique de la modernité et technoscepticisme}

La simplification réductrice du monde, sa banalisation et la recherche de son contrôle culminent dans toutes les entreprises de mécanisation. Ainsi, un des traits romantiques les plus saillants est la critique de la technologisation de la 
société, accusée d'une part - et encore une fois - d'aliéner les individus en les rendant esclaves de l'outil, et d'autre part de produire une aseptisation généralisée. Ainsi, dans ses versions les plus spontanées, le romantisme se présente comme un éloge de l'authenticité, de la diversité et de l'aléatoire face à une technologie artificielle, standardisée, et mécanique.

L'ensemble de ces traits sont remarquables chez nos six auteurs. Le couple artificialité/authenticité et le rejet de la mécanisation sont au cœur de la pensée des plus naturalistes (B. Charbonneau, A. Naess ou H. D. Thoreau), mais tout autant de celle des plus politistes:

la relation industrielle est réflexe conditionné, réponse stéréotypée de l'individu au message émis par un autre usager, qu'il ne connaîtra jamais, ou par un milieu artificiel, qu'il ne comprendra jamais. (Illich, 1973, p. 28)

La mécanisation, qui s’oppose à la liberté créatrice comme à la diversité célébrée par le romantisme, est également au cœur de la critique de ces écologies :

Pour l'écologie, c'est la différenciation organique, et non son contraire, la standardisation mécanique, qui permet de réaliser l'équilibre et l'harmonie dans la nature, la société et par suite, dans le comportement. (Bookchin, 1976, p. 84)

\section{5b) Réoutiller la société}

Il faut toutefois noter que les critiques romantiques (du moins dans leur version «romantique révolutionnaire ») ne sont ni antimodernes, ni technophobes par principe : elles plaident, selon l'expression d'Henri Lefebvre, non pour un retour, mais pour un détour par le passé pour inventer un monde meilleur. Concernant le rapport aux technologies, leur problème n'est pas l'outil en luimême, mais ce pour quoi il est pensé, à savoir la productivité et non l'épanouissement humain individuel et collectif. Le romantisme ne s'oppose pas à la modernité, mais à une certaine forme de modernité, industrielle, productiviste, capitaliste, etc.

Les auteurs de notre corpus se défendent explicitement d'être passéistes ou de refuser tout progrès : "une société nouvelle doit naître de la réintégration de la nature dans notre vie et il est bien probable qu'elle ne ressemblera pas plus à un impossible Moyen Âge qu'à la machine à habiter de Le Corbusier » (Charbonneau, 1937, p. 58). I. Illich encourage de son côté « une diversité des modes de vie, qu'elle tienne davantage de la mémoire, c'est-à-dire de l'héritage du passé, ou de l'invention, c'est-à-dire d'une création à nouveaux frais» (Illich, 1973, p. 36). La thématique du «détour par le passé » est claire dans ces lignes. Par ailleurs, ce rapport de l'écologie romantique au progrès est particulièrement sensible dans le topos du «soi-disant progrès» : il s'agit de critiquer non pas le "Progrès » en lui-même, mais ce qu'un état de société néfaste appelle lui-même, à tort, «progrès». La désignation de cette forme de novlangue traverse tout notre corpus : 
" presque tout ce que mes voisins appellent le bien, je crois de toute mon âme que c'est le mal» (Thoreau, 2017, p. 19); «la coopération des bureaucrates du bienêtre et des bureaucrates de l'idéologie nous fera crever de "bonheur" " (Illich, 1973, p. 30); «l'idéal naturiste est en contradiction avec le mythe du Progrès» (Charbonneau, 1937, p.42). Par ailleurs, le rapport de l'écologie romantique à la modernité technique s'exprime de manière éclatante dans le titre anglais de l'article et des conférences qui ont précédé l'ouvrage d'I. Illich : re-tooling society. Il ne s'agit pas de refuser les outils, mais de s'interroger sur leurs effets et de réorienter leurs fins comme leurs moyens.

Le parcours qui précède nous a permis de montrer que le corpus des auteurs que nous avons sélectionnés est traversé par une forte topique romantique : critique de la modernité aliénante, de la bureaucratisation, de la toute-puissance de la raison instrumentale, de la standardisation, de la démesure, etc. ; et par opposition défense de la sagesse, de la vie à petite échelle, de la liberté créatrice, de la diversité, de la recherche d'harmonie, etc. On pourra néanmoins noter que la plupart se défendent d'être romantiques, mais il s'agit là d'un effet du flou définitionnel de ce terme et des connotations péjoratives qui peuvent lui être associées. Qu'ils soient personnalistes, transcendantalistes, anarchistes, marxiens ou décroissants, avec les différences et les frictions importantes que cela peut entraîner, chacun participe à diffuser la topique romantique dans le corpus idéologique de l'écologie politique.

Cependant, montrer la présence de mots et d'idées romantiques dans une partie du corpus idéologique écologiste ne saurait suffire à en faire un moteur de la contestation écologiste contemporaine. En revanche, le fait que cette topique se retrouve de manière particulièrement visible dans les commentaires plus spontanés d'individus a priori profanes sur des sites web tend à confirmer le caractère circulant et agissant de cette forme idéologique. La section qui suit s'attache ainsi à montrer le romantisme de la contestation contre les projets d'aménagement industriel.

\section{Le romantisme de la contestation contre les projets d'aménagement}

Les résultats que nous présentons ici sont issus d'un travail d'analyse plus complet que l'on pourra consulter ailleurs (Carbou, à paraître). Nous nous sommes intéressé aux discours d'opposition aux projets du Center Parc de Roybon et de la "ferme des mille vaches» dans la Somme. Les discours recueillis proviennent de trois sites de presse, sélectionnés pour leur trafic web important et leur caractère de références dans le paysage médiatique français : Le Monde.fr (110 commentaires), Le Figaro.fr (128 commentaires), et FranceTVinfo.fr (164 commentaires). Nous y avons recherché la présence et la récurrence 
(au moins trois occurrences chez des internautes - ou du moins des pseudonymes - différents) des topoï caractéristiques du romantisme politique. Nous présentons ci-dessous des verbatims 3 qui illustrent les cinq grands axes de la critique romantique que nous avons développés plus haut : désenchantement du monde, critique de l'utilitarisme gestionnaire, de l'hégémonie de la rationalité instrumentale, de la modernité technolâtre et enfin de l'immodération.

\section{1) Le sentiment de désenchantement}

Le désenchantement romantique s'exprime chez les internautes dans le sentiment que le vivant et la diversité sont étouffés par l'artificiel et l'uniforme. Cette position est présente dans la critique des «bétonneurs » (Denise, FTV) et du «bétonnage de la nature » (Didierv, FTV) d'une part et de l'industrialisation de l'élevage d'autre part : « on transforme ces bêtes en usines à lait [...] merci le progrès! ». Ce qui est en cause ici est bien le ternissement du monde («marre du béton qui est gris », LL, LM), l'inauthenticité ("Je préfère encore pointer au chômage que de me balader, déguisée en vahiné, dans une ridicule bulle tropicale des montagnes iséroises... », PC, LM) et la standardisation ("si c'est cela l'avenir autant faire des clones ou des robots... », Raimu 30, FTV; "Avez vous des enfants? Quel futur pour eux si tout est automatisés », BB, FTV). C'est ainsi un monde entièrement affadi que les internautes voient dans l'avènement d'une «agriculture standardisée, sans saveur, sans odeur. La nourriture sous-vide, uniformisée» (Reiko, FIG).

\section{2) Utilitarisme gestionnaire et réification}

Le romantisme attribue ce sentiment d'atonie à la gestion rationalisée qui transforme les êtres en simples rouages. Cette critique traverse également tout le corpus de commentaires : «Ce type de ferme est une ignominie. [...] Les postes sont standardisés pour diminuer les coûts. Les fermiers [...] veulent continuer à exercer un vrai métier et ne pas devenir un simple OS sur une ligne de montage. » (Robinhood1, FIG); «les textes qui l'organisent [ce mode d'élevage] utilisent un langage anodin, technocratique : on ne parle pas de vie et de souffrance des animaux, mais de millions de litres de lait, de tonnes de viande, de capacité. » (M.M., LM). Les «technocrates» et les "politiciens hors-sol» (Armoo, FTV) sont ainsi accusés de mettre en œuvre une insupportable réification du vivant : «on ne considère l'animal que comme une machine à produire et à faire de l'argent. [...] tout juste bon à nourrir des êtres sans goût, sans cœur et sans cervelle» (MINERVEoo1, FIG).

3. Nous citons notre corpus sans correction orthographique ni typographique et mentionnons le pseudonyme ou ses initiales et le site de provenance entre parenthèses (LM pour Le Monde.fr, FIG pour Le Figaro.fr et FTV pour FranceTVinfo.fr). 


\section{3) Le chiffre contre la beauté du monde}

Au-delà du système politico-économique, nous avons vu que la critique romantique visait l'hégémonie d'une certaine forme de rationalité (instrumentale, calculante, analytique) qui serait aveugle à la complexité du monde et de l'expérience humaine. Les internautes déplorent ainsi que de simples indicateurs chiffrés puissent mesurer la qualité d'une entreprise :

je regrette que des cerveaux «endurcis» n'envisagent l'avenir que par la rentabilité au détriment de tout un système qui n'a pas si mal fonctionné jusqu'à présent, d'une diversité des goûts, ou tout simplement de la beauté d'un champ avec des vaches paisibles au milieu... (JoLeCrabe, FTV)

Le «calcul purement économique» (LS, FTV) est mis en balance avec l'ineffable de l'expérience subjective : "Certes un Center Parcs va redynamiser une zone économiquement pas très folichonne, mais cette forêt est belle et précieuse, je m'y suis souvent baladée, j'y ai cueilli des champignons, tout-ça, tout-ça.» (Ortie, FTV). Le monde ne peut être saisi par une formule mathématique ou logique: "Malheureusement le mal être animal ne se vérifie pas par des examens vétérinaires. » (huron47005, FIG). Cette conception d'un monde à expérimenter plutôt qu'à analyser se retrouve de manière remarquable dans la manière qu'ont les internautes d'opposer aux arguments techniques des descriptions subjectivisées : «je veux continuer de voir des vaches paitrent quand je me proméne dans la campagne, et devoir m'arreter et couper le moteur de la voiture, pour laisser passer ces indolentes reines des prés quand c'est l'heure de la traite » (BR, FTV). Plus généralement, il est intéressant de noter l'association récurrente que font les internautes entre ferme-usine et critique du «scientifique » : "À mon avis, les scientifiques, qui ont imaginés cette industrialisation doivent se cacher, comme les derniers des imbéciles.» (JM, FTV).

\section{4) Contre une certaine modernité}

Un autre pilier de la critique romantique est le rejet d'une modernité vécue comme destructrice. Les réactions d'internautes marquent souvent leur inquiétude face au «progrès » que représentent les différents projets d'aménagement pour leurs défenseurs : «Elever 1000 vaches (dans des conditions de vie épouvantables) avec quelques salariés (au SMIC), c'est sans doute l'avenir, selon vous. » (Reiko, FIG); "[nous] serons victime de ce fameux progrès utopiste et mensonger» (Bernard, FTV). On remarque bien qu'il ne s'agit pas d'une technophobie ou d'un antimodernisme de principe, mais bien du sentiment de «spoliation lexicale» (Angenot, 1982) que nous avons déjà relevé dans notre précédent corpus : on ne critique pas le progrès en soi, mais ce que d'autres 
appellent indûment progrès. Par ailleurs, et dans un esprit semblable, on peut également retrouver chez les internautes le topos caractéristique du «détour par le passé»: «ne confondez pas nostalgie avec obligation de changer de cap» (Armoo, FTV).

\section{5) Mesure et démesure}

Enfin, on peut remarquer que les internautes rejettent le progrès qui leur est proposé, car ils y voient un avatar de la «société de technologies inutiles et de gaspillage » $(\mathrm{CC}, \mathrm{FIG})$. On retrouve ainsi très régulièrement la critique romantique de la démesure moderne. Les internautes stigmatisent des «projets surdimensionnés [et] pharaoniques » (mélusine, LM) qu’ils trouvent «monstrueux» (jacques7166, FIG) et fustigent leur immodération : «Une serre tropicale chauffée au milieu du Vercors, un concept aussi goinfre que les villes du golfe Persique!» (JB, LM); « et pourquoi pas une grande entreprise avec mille maçons ou mille charcutiers ou mille électriciens?» (flocon670, FTV). La dangerosité de l'immodération moderne est particulièrement soulignée par le topos de la «fuite en avant », récurrent dans notre corpus : «Ils sont donc contraints à une fuite en avant pour construire» (DH, LM) ; «fuite en avant, accélérateur pied au plancher!» (adoque, FTV). En contrepoint, on retrouve dans le corpus de nombreux appels à la protection des structures "à dimension humaine» (maupie, LM); à un sens commun de la retenue : "C'est quoi un "aménagement”, à part tout mettre sens dessus dessous faute du sens de l'esthétique et de la mesure...» (jimoo9, FIG); voire à une pauvreté éthique : «je préfère être pauvre et respectez la terre qui nous supporte...plutot que d'etre riche et de courrir après l'argent au mépris de tout» (you hou, FIG).

Ainsi donc, ce rapide survol de notre corpus de discours contestataires d'internautes nous permet de voir que la topique romantique est présente en son sein. Elle est, évidemment, loin d'être la seule, mais elle y est incontestablement présente et opérante.

\section{Conclusion : une topique écologiste sans Nature}

Nos analyses plaident ainsi en faveur de la reconnaissance de la critique romantique comme une composante agissante de la nébuleuse idéologique écologiste. Présente de manière saillante dans le corpus des précurseurs de l'écologie politique, elle apparaît tout aussi vivement dans les propos spontanés d'internautes qui s'inscrivent dans des contestations environnementales.

Aussi, le concept d'écologie romantique, avec la topique qui le caractérise, nous apparaît opératoire pour l'analyse de la dynamique idéologique de l'écologie politique. Il permet par exemple de comprendre pourquoi des 
contestations dites «écologistes» peuvent aussi bien s’élever contre des projets a priori écologiques (des éoliennes, des fermes solaires...) et pourquoi des problèmes apparemment locaux (les projets d'aménagement du territoire) attirent des opposants de zones géographiques éloignées : une partie de ces résistances sont en fait moins écologiques que romantiques en cela qu'elles combattent avant tout la modernité capitaliste industrielle. De plus, en tant qu'il motive une certaine forme d'écologie, le romantisme permet d'expliquer des dissensions internes à la nébuleuse écologiste (écologie libertaire vs autoritaire par exemple) ou encore de comprendre - et éventuellement de contester - les qualificatifs critiques que suscitent parfois les mouvements écologistes (réactionnaire, antidémocratique, antihumaniste, passéiste, protofasciste, etc., voir Flipo, 2014).

Par ailleurs, et pour finir, il est frappant de remarquer la quasi-absence de référence à la nature dans la logique de la pensée romantique que nous décrivons. En résumé, cette forme d'écologie considère que la constitution d'une société non aliénante pour l'être humain doit aller de pair avec une vie harmonieuse avec la biosphère. Si elle mobilise largement la thématique de la nature, la défense de la nature (de toute manière indéfinissable dans cette pensée de la totalité) n'est pas pour elle un objectif en soi.

Il apparaît en fait que la nature n'est, pour l'écologie romantique, rien de plus - mais rien de moins - qu'un prétexte ou un symbole. Un prétexte, d'abord, car la protection de la nature s'oppose de fait à la volonté de conquête et d'illimitation inhérente au capitalisme industriel : refuser l'exploitation des forêts, des gisements d'énergie fossile ou l'utilisation de pesticides, c'est mettre un coup d'arrêt à l'ensemble de l'entreprise industrielle moderne.

Un symbole ensuite, car l'imaginaire que l'on peut attacher à la nature contraste radicalement avec celui de la modernité vue d'un œil romantique : la nature est imprévisible là où la bureaucratie est une obsession du contrôle ; elle est l'objet d'émotions et de valeurs non marchandisables là où le capitalisme cherche justement à transformer toutes les sphères de la vie en marché ; elle représente le sauvage et l'authentique là où l'artificialisation et la technologie découlent de notre système économique; elle fonctionne de manière (éco-)systémique là où la rationalité gestionnaire voudrait tout découper en secteurs distincts; elle est le royaume du lien et de l'interdépendance, quand leur dissolution est le préalable à la colonisation néolibérale, etc.

L'usage du concept d'écologie romantique nous semble ainsi à même de saisir la spécificité d'une écologie qui ne doit être confondue ni avec un simple environnementalisme, ni avec un anticapitalisme productiviste. D'un point de vue anthropologique, c'est également un outil qui nous permet de penser l'une des formes culturelles les plus actives et les moins nommées de notre espace public contemporain. 


\section{Corpus}

BоoкCHIN Murray, 1976, «Écologie et pensée révolutionnaire », dans M. Bookchin, Pour une société écologique, H. Arnold et D. Blanchard trad., Paris, Christian Bourgois, p. 70-96.

Charbonneau Bernard, 1937, «Le sentiment de la nature, force révolutionnaire », Journal intérieur des groupes personnalistes du Sud-Ouest, p. 1-53.

Gorz André, 1977, Écologie et liberté, Paris, Galilée.

ILlich Ivan, 1973, La convivialité, L. Giard et V. Bardet trad., Paris, Seuil.

NAESS Arne, 2008, Écologie, communauté etstyle de vie, C. Ruelle trad., Paris, Éditions MF. THOREAU Henry David, 2017, Walden, B. Matthieussent trad., M. Granger éd., Marseille, Le mot et le reste.

\section{Références}

ANGENOT Marc, 2012, Le rationnel et le raisonnable: sur un distinguo de Chaïm Perelman, Montréal, Chaire James McGill d'étude du discours social.

- 2006, «Théorie du discours social : notions de topographie des discours et de coupures cognitives », COnTEXTES. Revue de sociologie de la littérature, n ${ }^{\circ} 1$, https:// journals.openedition.org/contextes/51 (consulté le 16 novembre 2018).

- 1991, "Les idéologies ne sont pas des systèmes ", Cahiers Ferdinand de Saussure, n० 45 , p. 51-76.

- 1982, La parole pamphlétaire : contribution à la typologie des discours modernes, Paris, Payot.

AUDIER Serge, 2017, La société écologique et ses ennemis : pour une histoire alternative de l'émancipation, Paris, La Découverte.

Bourg Dominique, PAPAUX Alain, 2015, Dictionnaire de la pensée écologique, Paris, PUF.

CARBou Guillaume, à paraître, «Le romantisme de la contestation contre les "GPII" dans les commentaires de presse en ligne", dans Les paroles militantes dans les controverses environnementales : constructions, légitimations, limites, V. Carlino et M. Stein éd., Nancy, Presses universitaires de Lorraine.

- 2015, Les médiations symboliques à l'œuvre dans les débats de société : l'exemple de l'accident nucléaire de Fukushima dans les commentaires d'actualité sur le web, thèse sous la direction de M. Coulomb-Gully et A. Zinna, Université Toulouse-JeanJaurès.

FLIPO Fabrice, 2014, Nature et politique : contribution à une anthropologie de la modernité et de la globalisation, Paris, Éditions Amsterdam.

GUEST Bernard, 2012, "Le cosmos libertaire de Thoreau et Reclus, un ordre sans pouvoir », dans Philosophies de l'anarchie : théories libertaires, pratiques quotidiennes et ontologie, J.-C. Angaut, D. Colson et M. Pucciarelli éd., Lyon, Atelier de création libertaire, p.11-31.

GUSDORF Georges, 1982, Fondements du savoir romantique, Paris, Payot.

LöWr Michael, BLECHMAN Max, 2004, "Qu'est-ce que le romantisme révolutionnaire? », Europe, revue littéraire mensuelle, $n^{\circ} 900$, p. 3-5.

LöWY Michael, SAYRE Robert, 2010, Esprits de feu : figures du romantisme anti-capitaliste, Paris, Éditions du Sandre. 
- 1992, Révolte et mélancolie : le romantisme à contre-courant de la modernité, Paris, Payot.

QUÉRÉ Louis, 1992, "L'espace public: de la théorie politique à la métathéorie sociologique», Quaderni, n¹8, p.75-92.

SAYRE Robert, LöwY Michael, 1984, «Figures du romantisme anti-capitaliste : une tentative de typologie ", L'Homme et la société, no 73-74, p.147-172.

- 1983, "Figures du romantisme anti-capitaliste», L'Homme et la société, n 69-70, p. 99-121.

\section{Résumé / Abstract / Compendio}

\section{La topique romantique dans les discours de l'écologie politique}

Dans cet article, notre objectif est de montrer que ce que nous appellerons romantisme est un des moteurs idéologiques de l'écologie. Le terme romantisme doit être pris ici non dans une acception simplement littéraire ou artistique, mais dans le sens politique : le romantisme est une protestation culturelle contre la modernité industrielle et capitaliste fondée sur le rejet des différents processus d'aliénation qu'elle produit. Nous proposons de définir cinq grands axes topiques de cette forme idéologique, puis nous montrons leur présence dans deux corpus : d'une part chez six auteurs considérés comme des penseurs inspirateurs de l'écologie politique (Bernard Charbonneau, André Gorz, Ivan Illich, Henry David Thoreau, Arne Naess et Murray Bookchin) et d'autre part dans un corpus de réactions contestataires d'internautes à des articles de presse en ligne traitant de grands projets d'aménagement du territoire. Nous entendons ainsi montrer comment le romantisme, déjà présent de manière assez canonique dans le corpus philosophique de l'écologie se retrouve également, de manière plus diffuse, dans les contestations environnementalistes profanes.

Mots-clés: romantisme, écologie, analyse du discours, formes culturelles, projets d'aménagement du territoire

\section{Political ecology discourses and romanticism}

This paper shows that what is called romanticism is an ideological engine that fuels contemporary ecology. Romanticism in this sense does not simply apply to arts but to a wide political movement: romanticism is a cultural reaction against industrial and capitalist modernity and is based on the critique of the various alienation processes this modernity generates. The present paper defines the five core axes of this critique and then highlights their presence in two separate corpuses. The first corpus consists of six texts from six authors widely seen as inspirationnal for political ecology (Bernard Charbonneau, André Gorz, Ivan Illich, Henry David Thoreau, Arne Naess and Murray Bookchin). The second corpus is composed of critical comments from internet users on online press articles dealing with regional development projects. These two different corpuses shed light on the fact that romantic ideology fuels the scholarly literature on political ecology as well as the more profane environmental protests.

Keywords: romanticism, ecology, discourse analysis, cultural forms, regional development projects 


\section{El tópico romántico en el discurso de la ecología política}

En este artículo, nuestro propósito es mostrar que lo que llamamos romanticismo constituye uno de los motores ideológicos de la ecología. La palabra romanticismo no tiene que ser percibida en su dimensión meramente literaria o artística, pero en su sentido político: el romanticismo es un protesta cultural contra la modernidad industrial y capitalista basada sobre el rechazo de los diferentes procesos de alienación que produce. Proponemos definir cinco grandes ejes tópicos de esta forma ideológica localizados dentro de dos corpus: por un lado, en seis autores considerados como los inspiradores de la ecología política (Bernard Charbonneau, André Gorz, Ivan Illich, Henry David Thoreau, Arne Naess et Murray Bookchin) por el otro, en un corpus de reacciones contestatarias de los usuarios de internet en los artículos de la prensa online que tratan de los grandes proyectos de desarroyo del territorio. Se piensa demostrar de esa manera como el romanticismo, ya presente de manera bastante canónica en el corpus filosófico de la ecología, se vuelve a encontrar, de manera más difusa, en las contestaciones ambientales más corrientes.

Palabras claves: romanticismo, ecología, análise del discurso, formas culturales, proyectos de desarroyo del territorio 
